\title{
Based on the Gray GM $(1,1)$ Prediction Model of Pig Disease
}

\author{
Yanying $\mathrm{Ma}^{1, \mathrm{a}^{*}}$, Xiuzhen $\mathrm{Li}^{1}$ and Xue Wang ${ }^{2}$ \\ ${ }^{1}$ Jilin Engineering Normal University .Changchun; China; \\ ${ }^{2}$ College of Mathematics and Computation Science Fuyang Teachers' college. Fuyang; China; \\ aarnold0110@sina.com
}

Keywords: Gray prediction model; Disease prediction; $G M(1,1)$ model

\begin{abstract}
With the growth of social economy, more and more high to the requirement of animal husbandry. With the brisk development of pig raising production, we have realized that how to improve the effectiveness of pig raising, disease warning and prevention is of great significance. In this research, by applying GM $(1,1)$ model of gray system theory, we try to forecast the pig disease based on the mortality rate of pig disease in a Jilin Provincial village. From the results, we can see the error between predicted values and measured values is very small and the fitting effect is excellent, which indicate that the method to predict pig disease is feasible, meanwhile, it will play a great role in health epidemic and disease prevention.
\end{abstract}

\section{The Theory and Method of Modeling Gray GM $(1,1)[1][8]$}

Date Processing. Let the sequence of primary data are nonnegative and discrete, $x(t)=\{x(1), x(2), \ldots, x(n)\}$, add the elements in which for once, we get the data:

$$
Y(t)=\sum_{t=1}^{t} x(i), t=1,2, \cdots, n
$$

Then finding the mean value of the data $Y(t)$ can we generate the following:

$$
Z(t)=\frac{1}{2}[Y(t)+Y(t-1)], t=2,3, \cdots, n \text {. }
$$

The Building of Model GM $(1,1)$. Formulating the first order differential equation of $Y(t)$ :

$$
\frac{d \mathrm{Y}(t)}{d t}+a Y(t)=u
$$

In which, $u$ and $a$ satisfying:

$$
Y(t)=\left(x(1)-\frac{u}{a}\right) e^{-a(t-1)}+\frac{u}{a} .
$$

Estimate the vectors of parameters $u$ and $a$ by least square technique, and the expressions of them obtained by matrix operations are the followings :

$$
\begin{gathered}
a=\frac{1}{D}\left\{(n-1)\left[-\sum_{t=2}^{n} X(t) Z(t)\right]+\left[\sum_{t=2}^{n} Z(t)\right]\left[\sum_{t=2}^{n} \mathrm{X}(t)\right]\right\} \\
u=\frac{1}{D}\left\{\left[\sum_{t=2}^{n} Z(t)\right]\left[-\sum_{t=2}^{n} X(t) Z(t)\right]+\left[\sum_{t=2}^{n} Z^{2}(t)\right]\left[\sum_{t=2}^{n} \mathrm{X}(t)\right]\right\} \\
\mathrm{D}=(n-1)\left[\sum_{t=2}^{n} Z^{2}(t)\right]-\left[\sum_{t=2}^{n} Z(t)\right]^{2}
\end{gathered}
$$


Take them into the differential equation with separable variable we can get the estimated values of $Y(t)$ and $\mathrm{X}(t)$ as follows:

$$
\begin{aligned}
& \hat{Y}(t)=\left(\mathrm{X}(1)-\frac{u}{a}\right) e^{-a(t-1)}+\frac{u}{a} \\
& \hat{\mathrm{X}}(\mathrm{t})=\hat{\mathrm{Y}}(\mathrm{t})-\hat{\mathrm{Y}}(\mathrm{t}-1), \mathrm{t}=2,3, \cdots, n
\end{aligned}
$$

Check the Fitting Effect of Sequences $\hat{X}(t)$ and $X(t)$.If the fitting accuracy of the two is good, it can be used to extrapolate prediction; otherwise, it can't be used to extrapolate prediction directly unless being modified. To determine the reliability, we can use average relative error, posterior error ratio and minor error probability to check.

The average relative error is:

$$
\bar{e}=\frac{\sum_{t=2}^{n}|\hat{X}(t)-X(t)|}{\sum_{t=2}^{n} X(t)} \times 100 \%
$$

Let the error $\varepsilon(t)$ be:

$\varepsilon(t)=X(t)-\hat{X}(t), t=2,3, \cdots, n$.

Calculate the posterior error ratio $\mathrm{C}$ and minor error probability $\mathrm{P}$ as:

$$
\mathrm{C}=\frac{\mathrm{S}_{2}}{\mathrm{~S}_{1}},
$$

$P=P\left\{|\varepsilon(t)-\bar{\varepsilon}|<0.685 S_{1}\right\}$,

Where

$$
\begin{aligned}
& S_{1}^{2}=\frac{1}{n} \sum_{t=1}^{n}[X(t)-\bar{X}]^{2} \\
& \bar{X}=\frac{1}{n} \sum_{t=1}^{n} X(t) \\
& S_{2}^{2}=\frac{1}{n} \sum_{t=1}^{n}[\varepsilon(t)-\bar{\varepsilon}]^{2} \\
& \bar{\varepsilon}=\frac{1}{n} \sum_{t=1}^{n} \varepsilon(t)
\end{aligned}
$$

Judge the fitting degree of gray sequence according to the reference table on accuracy-checking grade. 
Table 1 Judge the fitting degree of the prediction model GM $(1,1)$

\begin{tabular}{|c|c|c|}
\hline Accuracy Grade of the model & P value & C value \\
\hline Good & $>0.95$ & $<0.35$ \\
\hline Qualification & $>0.80$ & $<0.50$ \\
\hline Reluctance & $>0.70$ & $<0.65$ \\
\hline Disqualification & $\leq 0.70$ & $\geq 0.65$ \\
\hline
\end{tabular}

Extrapolate Prediction. A good fitting degree means the effect of the prediction model is feasible, so we can do extrapolate prediction by the following formula:

$$
\hat{\mathrm{X}}(\mathrm{t})=\hat{\mathrm{Y}}(\mathrm{t})-\hat{\mathrm{Y}}(\mathrm{t}-1), \mathrm{t}=n+1, n+2, \cdots, n+k
$$

\section{Examples}

\section{Resource of Data Information.}

Table 2 Pig disease mortality rate (1\%) in a Jilin Provincial village (2009-2015)

\begin{tabular}{|c|r|r|r|r|r|r|c|}
\hline order & 1 & 2 & 3 & 4 & 5 & 6 & 7 \\
\hline year & 2009 & 2010 & 2011 & 2012 & 2013 & 2014 & 2015 \\
\hline $\begin{array}{c}\text { Real disease death } \\
\text { rate (\%) }\end{array}$ & 2.88 & 2.36 & 2.62 & 2.24 & 2.14 & 2.06 & 2.03 \\
\hline $\begin{array}{c}\text { Predicted disease } \\
\text { death rate (\%) }\end{array}$ & 2.88 & 2.5073 & 2.3972 & 2.2926 & 2.1924 & 2.0962 & 2.0023 \\
\hline
\end{tabular}

By using the real disease death rate from 2009 to 2015 in a Jilin Provincial village in table 2 and applying the GM $(1,1)$ model, we build the corresponding prediction model. From the formulas (5), (6), (7), we find that $\hat{a}=0.0448, \hat{u}=2.6699$ and $D=522.7839$ Take the above-mentioned values on formula (8), the prediction equation will be acquired as follows:

$$
\hat{Y}(t)=-57.1247 e^{-0.0449(t-1)}+59.54
$$

\section{Discussion}

Gray system theory is an interdisciplinary subject for researching indetermination and it has made much success in many practical fields since it appeared in 1982, there is no exception in disease monitoring and warning. The model of gray prediction is simple and convenient to calculate with less concerning influence factors. Depending on systematical known information to accumulate irregular data to generate a relatively more regular generating sequence by gray modular, then we can build differential dynamic time sequence model, process the gray information and improve the Whitening degree of gray model by a certain rule, accordingly, we will reach the goal of further prediction on the 
base of disclosing the internal features and regularities of matters, Compared with traditional model of mathematical statistics, the model, can be used as an effective tool, has many good qualities in predicting diseases, such as, it needs less amount of samples, no typical probability distribution and less calculations. From the results, we can see the error between predicted values and measured values is very small and the fitting effect is excellent, which indicate that the method to predict pig foot and mouth disease is feasible, meanwhile, it will play a great role in preventing disease.

\section{Acknowledgements}

Jilin provincial department of education project (2016): research and applications of mineral prediction methods based on grey theory.

\section{References}

[1] Zhuyan Shao, Chunmei Wang. Application of the grey system GM $(1,1)$ forecast model on predictive diseases. [J] .Journal of Chinese Journal of Hospital Stastics, Sep.2003, Vol. 10, No. 3.

[2] Tao Liu. Application of Grey of the Series Forecasting Model of the Population Mortality [J]. Journal of Yunyang Teachers' College. Jun.2004, Vol.24, No.3.

[3] Xiangyun Li, Xiaodong Liu, Limin Ma and Rui Jing. Application of grey theory model GM $(1,1)$ on forecasting infant mortality rate [J]. Chinese Journal of Hospital Statistic. Mar. 2009, Vol.16, No.1.

[4] Wujian Li, Xianming Yi and Weiwei Gu. Predicting the Epidemic Tendency of Malaria in a City by Gray Model [J]. Journal of Yangtze University, Jun. 2006, Vol. 3, No. 2: 282 285.

[5] Xia Zhou, Hong Qiiu and Peng Wang. Method for Mathematic Modeling of Grey Prediction and its Application in Medical Science [J]. Journal of Mathematical Medicine. 